\title{
Análisis correcto de los cementos por medio de la absorción atómica
}

\section{INTRODUCCION}

En trabajos anteriores (1), (2) y (3) se han subrayado las posibilidades que ofrece la espectrofotometría de absorción atómica (E.A.A.) para el análisis de los silicatos industriales (cementos, escorias, crudos de fabricación de cementos, refractarios, etc.).

En particular son del mayor interés los análisis de los cementos; el conjunto de los resultados obtenidos nos permite proponer un método que es conveniente para el análisis de todos los cationes del cemento a partir de una sola disolución.

El examen estadístico de nuestros resultados revela que la precisión de las valoraciones efectuadas con este método es totalmente satisfactoria para todos los elementos, excepto para el calcio. Recordemos que la valoración de calcio en los cementos ha sido objeto de numerosas investigaciones: se busca mejorar la precisión en los análisis mediante la determinación del calcio por la E.A.A. Bonomi, Francardi y Missazza (4) han reexaminado el problema del análisis de los cementos mediante la E.A.A. Estos autores obtienen una dispersión satisfactoria $(\sigma=0,1)$ en la dosificación de calcio, pero esta dosificación se hace después de la precipitación de la sílice y lavados del precipitado para recuperar los cationes arrastrados en el curso de la precipitación del $\mathrm{SiO}_{2}$. Por otra parte, el método de Bonomi y otros (4) requiere demasiada minuciosidad para que pueda ser aplicado corrientemente en un laboratorio industrial: por ejemplo, estos autores estabilizan térmicamente todas sus soluciones a $21^{\circ} \mathrm{C}$; además, recurren a una segunda disolución para valorar la sílice.

Recientemente, Voinovitch y sus colaboradores (5) han dado a conocer que el empleo de los espectrofotómetros de doble haz permiten obtener resultados bastante interesantes para la dosificación del calcio en los cementos.

El examen estadístico de un número muy amplio de análisis del calcio con la E.A.A. ha permitido a Voinovitch (6) encontrar una desviación típica digna de confianza para la dosificación de $\mathrm{Ca}$; por otra parte, este autor ha puesto en evidencia el importante papel de las condiciones experimentales.

Teniendo en cuenta los progresos adquiridos desde nuestros primeros trabajos (1), hemos tratado de mejorar nuestro método, sobre todo en lo que se refiere a la dosificación de calcio. Quisiéramos insistir en el hecho de que las mejoras han sido aportadas conservando las ventajas esenciales del método, es decir, el empleo de una sola disolu- 
ción para dosificar los cationes del cemento. Más adelante expondremos nuestro método "mejorado", que tiene una precisión satisfactoria para todos los elementos (incluido el calcio).

\section{Observaciones sobre el empleo de la E.A.A. para el análisis cuantitativo de los silicatos}

La precisión del análisis de los cementos u otros materiales similares por medio de la E.A.A. depende de los factores siguientes:

I. La preparación de la disolución.

II. La supresión correcta de las interferencias químicas, iónicas y espectrales.

III. La elección de las soluciones-patrón.

IV. El empleo de un espectrofotómetro de alta calidad, de una gran estabilidad (doble haz), $\mathrm{y}$, sobre todo, la perfecta puesta a punto de las condiciones experimentales.

\section{I) LA PREPARACION DE LA DISOLUCION}

Salvo algunos casos excepcionales, el análisis de sólidos por medio de la E.A.A. se hace a partir de una disolución, es decir, que la preparación de la disolución constituye la primera etapa del análisis por medio de la E.A.A.; un buen método de preparación de la disolución debe permitir el ataque rápido e integro de la muestra por reactivos que no alteren la dosificación de los elementos de la muestra.

El método de disolución que hemos adoptado se basa en atacar al cemento con una mezcla de $\mathrm{HCl}+\mathrm{HF}$; a continuación se describe brevemente.

Se pesa (con un error de $\pm 0,1 \mathrm{mg}$ ) alrededor de $1.500 \mathrm{~g}$ de cemento en un vaso de P.C.V.; progresivamente se añaden $20 \mathrm{ml}$ de agua destilada, se agita el contenido del vaso con ayuda de un agitador de P.C.V. con el fin de obtener una suspensión homogénea. Después de homogeneizado, se añaden $20 \mathrm{ml}$ de $\mathrm{HCl}$ concentrado $(d=1,116)$. Se aumenta el volumen hasta $150 \mathrm{ml}$ con agua destilada.

Entonces se añaden $3 \mathrm{ml}$ de $\mathrm{HF}$ concentrado (40\%); el contenido del vaso se agita durante 2 minutos, seguidamente se añaden $25 \mathrm{ml}$ de una solución saturada $(5 \%)$ de $\mathrm{H}_{3} \mathrm{BO}_{3}$. La disolución se filtra con un filtro Millipore de $1,2 \mu$.

El filtro se lava previamente con unos $75 \mathrm{ml}$ de una disolución de $\mathrm{HCl}$ al $5 \%$, y a continuación con $100 \mathrm{ml} \mathrm{de} \mathrm{H}_{2} \mathrm{O}$ destilada. El filtrado se transvasa cuantitativamente a un matraz aforado de $500 \mathrm{ml}$; el volumen de la disolución de cemento se enrasa con $\mathrm{HCl}$ al 5 por ciento.

La disolución de cemento se transvasa inmediatamente después del ajuste del volumen a un recipiente de P.C.V.

Precisamente a partir de esta solución es con la que dosificaremos los diez óxidos siguientes: $\mathrm{CaO}, \mathrm{SiO}_{2}, \mathrm{Al}_{2} \mathrm{O}_{3}, \mathrm{Fe}_{2} \mathrm{O}_{3}, \mathrm{MgO}, \mathrm{Na}_{2} \mathrm{O}, \mathrm{K}_{2} \mathrm{O}, \mathrm{Mn}_{2} \mathrm{O}_{3}, \mathrm{SrO}$ y $\mathrm{TiO}_{2}$.

Es evidente que se debe diluir esta solución de diferente forma según el elemento a determinar. 
Esta técnica de disolución tiene dos ventajas fundamentales:

1) No introduce ningún catión (aparte de los bien conocidos elementos del cemento) durante la disolución.

2) El proceso de disolución es rápido, dura alrededor de un cuarto de hora (12 minutos exactamente). La disolución del cemento portland se realiza a temperatura ambiente; el residuo obtenido por este método es, en general, inferior al que se obtiene en las disoluciones por los procedimientos clásicos (ataque con clorhídrico).

Este método de disolución es también conveniente para la disolución de los cementos que contienen escorias.

\section{II) LAS DIVERSAS INTERFERENCIAS Y SU ELIMINACION}

Las interferencias químicas entre los elementos que entran en una disolución de un cemento se conocen perfectamente (3), (7), (8) y (9). Hemos estudiado la formación de los compuestos que tienen puntos de fusión bastante elevado cuando se evapora una disolución de cemento en la llama.

No parece sea necesario desarrollar aquí con detalle el mecanismo de la formación de los compuestos refractarios: lo que particularmente le interesa al analista es el medio de suprimir las interferencias.

El método de disolución que proponemos conduce a practicar la dosificación en una matriz que contiene complejos fluobóricos. Bernas (10) ha demostrado, y nosotros lo hemos confirmado también, que una matriz que contenga $\mathrm{HCl}-\mathrm{HF}-\mathrm{H}_{3} \mathrm{BO}_{3}$ atenúa en un elevado grado las interferencias químicas. Sin embargo la supresión de las interferencias químicas no es total, y esta es la razón para que nosotros utilicemos lantano. Este elemento es extremadamente útil en la valoración de ciertos cationes de los cementos por medio de la E.A.A.

Se han efectuado ensayos preliminares con el fin de encontrar la concentración óptima de La: por concentración óptima, entendemos el contenido mínimo de La que suprime las interferencias químicas. Un exceso de lantano hace la señal inestable; dicho de otro modo, se puede tener una precisión mediocre provocada por un elevado contenido en La.

Para la valoración de ciertos elementos $(\mathrm{K}, \mathrm{Na}, \mathrm{Sr})$ se deben suprimir las perturbaciones debidas a la ionización de los átomos en la llama. Para eliminar las ionizaciones hemos recurrido al ClCs como tampón espectral. Para el cloruro de cesio, igualmente hemos investigado la concentración óptima (ver el esquema de las disoluciones). Recordemos, además, que el ClCs tiene un efecto beneficioso sobre la sensibilidad del titanio.

\section{III) METODOS DE DOSIFICACION. DISOLUCIONES-PATRON}

Efectuamos nuestras valoraciones según el método de "ajuste". En este método la muestra se acota con dos disoluciones-patrón (cuyas composiciones se conocen con exactitud). La figura 1 muestra de una manera esquemática el ajuste de la muestra desconocida para los patrones I y II, siendo $D_{I}, D_{\text {II }}$ y D las densidades ópticas respectivas del patron I, 
el patrón II y la desconocida, y $\mathrm{C}_{\mathrm{e}}, \mathrm{C}_{\mathrm{e} I \mathrm{y}}$ y $\mathrm{C}$, la concentración (expresada en $\mu \mathrm{g} / \mathrm{ml}$ ) del elemento a valorar en el patrón I, el patrón II y la desconocida. La siguiente ecuación nos permite calcular $\mathrm{C}$ :

$$
\mathrm{C}=\mathrm{C}_{\mathrm{e} I}+\left[\frac{\left(\mathrm{C}_{\mathrm{eII}}-\mathrm{C}_{\mathrm{e}} \mathrm{I}\right) /\left(\mathrm{D}-\mathrm{D}_{\mathrm{I}}\right)}{\mathrm{D}_{\mathrm{II}}-\mathrm{D}_{\mathrm{I}}}\right]
$$

Para tener una mejor precisión es necesario que las concentraciones del elemento a valorar en las disoluciones-patrón sean tan aproximadas como sea posible a la disolución problema. Por otra parte, para que la exactitud de la valoración no sufra el efecto de matriz, la composición química de las disoluciones-patrón también debe ser tan parecida como sea posible a la de la disolución a analizar.

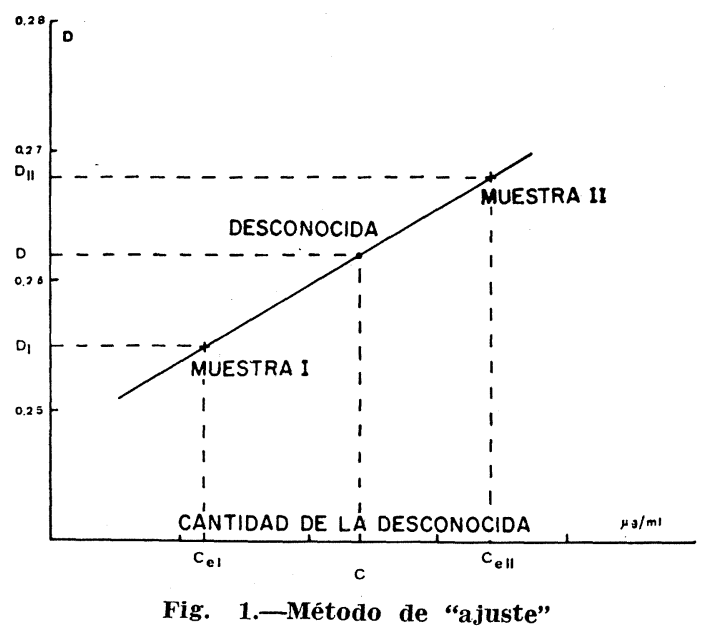

tos a valorar. Seguidamente resumiremos las características de estas disoluciones-patrón. Cada disolución-patrón contiene los diez óxidos siguientes: $\mathrm{CaO}, \mathrm{SiO}_{2}, \mathrm{Al}_{2} \mathrm{O}_{3}, \mathrm{Fe}_{2} \mathrm{O}_{3}$, $\mathrm{MgO}, \mathrm{Na}_{2} \mathrm{O}, \mathrm{K}_{2} \mathrm{O}, \mathrm{Mn}_{2} \mathrm{O}_{3}, \mathrm{SrO}$ y $\mathrm{TiO}_{2}$. Aunque la disolución sea preparada a partir de productos espectroscópicamente puros es, sin embargo, idéntica a una que se obtenga al realizar la disolución de un cemento, aplicando nuestro método.

En este caso, no hay prácticamente efecto de matriz.

Hemos preparado seis disoluciones-patrón que permiten el análisis de seis elementos en los cementos. Estas disoluciones-patrón deben ser diluidas en el momento de ser empleadas. Las hechas para ser almacenadas tienen un período de conservación bastante aceptable. Los títulos de estas disoluciones permanecen perfectamente constantes hasta por lo menos un año después de haber sido preparadas (nosotros hemos podido comprobarlo, incluso sobrepasada esta fecha). Es decir, que en los laboratorios de control se pueden preparar una vez al año una serie de disoluciones-patrón. Para analizar los cementos industriales belgas, tanto los portland como los metalúrgicos, hemos empleado seis disoluciones-patrón: la composición relativa de éstas, en óxidos, puede verse en la tabla 1. 
T A B L A 1

\begin{tabular}{|c|c|c|c|c|c|c|c|c|c|c|}
\hline $\begin{array}{c}\text { Disoluciones } \\
\text { patrón }\end{array}$ & $\mathbf{C a O}(*)$ & $\mathbf{S i O}_{2}$ & $\mathbf{A l}_{2} \mathbf{O}_{3}$ & $\mathbf{F e}_{2} \mathbf{O}_{3}$ & $\mathbf{M g O}$ & $\mathbf{N a}_{2} \mathbf{O}$ & $\mathbf{K}_{2} \mathbf{O}$ & $\mathbf{M n}_{2} \mathbf{O}_{3}$ & Sro & $\mathbf{T H O}_{2}$ \\
\cline { 1 - 2 } & 68 & 17 & 3 & 7 & 0,5 & 0,10 & 1,20 & 0,05 & 0,05 & 0,05 \\
2 & 66 & 19 & 4 & 5 & 1 & 0,20 & 0,90 & 0,10 & 0,10 & 0,10 \\
3 & 64 & 21 & 5 & 4 & 2 & 0,30 & 0,60 & 0,20 & 0,15 & 0,20 \\
4 & 62 & 23 & 6 & 3 & 3 & 0,40 & 0,45 & 0,30 & 0,2 & 0,30 \\
5 & 60 & 25 & 8 & 2 & 4 & 0,50 & 0,30 & 0,40 & 0,25 & 0,40 \\
6 & 58 & 27 & 10 & 1 & 5 & 0,60 & 0,15 & 0,60 & 0,3 & 0,55 \\
\hline
\end{tabular}

(*) Las cantidades de los óxidos están expresadas en \%.

Después del análisis del cemento, se deben diluir las disoluciones-patrón para efectuar la valoración. Las diluciones de las disoluciones-patrón y de los cementos portland son idénticas. En la figura 2 se representa el esquema de la dilución necesaria para la valoración de los diferentes elementos; las disoluciones-patrón se diluyen de la misma manera.

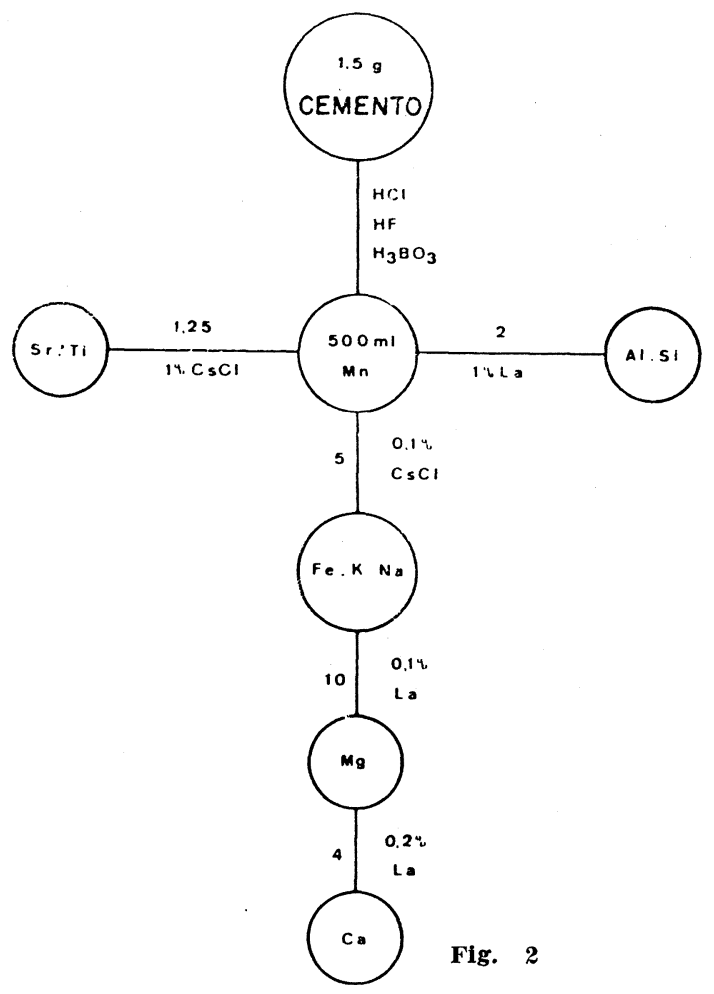

En la tabla 2 se indican las concentraciones (expresadas en $\mu \mathrm{g}$ de óxido/ml) de los diez elementos en las disoluciones-patrón después de efectuadas las disoluciones.

Se ve, por ejemplo, que la concentración de $\mathrm{MgO}$ de las disoluciones varía de 0,3 a $3 \mu \mathrm{g} /$ $/ \mathrm{ml}$. Nosotros hemos establecido para todos los elementos las curvas resultantes de la representación de la densidad óptica, $\mathrm{D}$, en función de la concentración. La figura 3 representa las variaciones de $\mathrm{D}$ en función de las concentraciones de $\mathrm{MgO}$ en las seis disoluciones-patrón. La figura 4 es una curva de trabajo representada con expansión de escala. Para cementos que contengan cantidades muy débiles de $\mathrm{MgO}$ conviene trabajar con expansión de escala.

TAB L A 2

\begin{tabular}{|c|c|c|c|c|c|c|c|c|c|c|}
\hline $\begin{array}{c}\text { Disoluciones } \\
\text { patrón }\end{array}$ & $\mathbf{C a O}$ & $\mathrm{SiO}_{2}$ & $\mathbf{A l}_{2} \mathbf{O}_{3}$ & $\mathbf{F e}_{2} \mathbf{O}_{3}$ & Mgo & $\mathbf{N a}_{2} \mathbf{O}$ & $\mathbf{K}_{2} \mathbf{O}$ & $\mathbf{M n}_{2} \mathbf{O}_{3}$ & Sro & TrO $_{2}$ \\
\hline 1 & 10,2 & 256,0 & 45,0 & 42,0 & 0,3 & 0,55 & 7,3 & 1,55 & 1,75 & 1,25 \\
\hline 2 & 9,9 & 286,0 & 60,0 & 30,0 & 0,6 & 1,1 & 5,4 & 3,10 & 2,9 & 2,5 \\
\hline 3 & 9,6 & 315,0 & 75,0 & 24,0 & 1,2 & 1,7 & 3,7 & 6,2 & 4,1 & 4,9 \\
\hline 4 & 9,3 & 345,0 & 90,0 & 18,0 & 1,8 & 2,2 & 2,7 & 9,3 & 5,2 & 7,4 \\
\hline 5 & 9,0 & 378,0 & 120,0 & 12,0 & 2,4 & 2,7 & 1,9 & 12,4 & 6,4 & 9,8 \\
\hline 6 & 8,7 & 405,0 & 150,0 & 6,0 & 3,0 & 3,3 & 0,8 & 18,6 & 7,7 & 13,5 \\
\hline
\end{tabular}


En las curvas $D=f(C)$ (siendo $C$ la concentración expresada en $\mu g$ de óxido/ml) hay una parte lineal. El límite superior de concentración de esta parte rectilínea varía de un elemento a otro; a esta parte rectilínea se la llama "Linear Working Range" (L.W.R.).

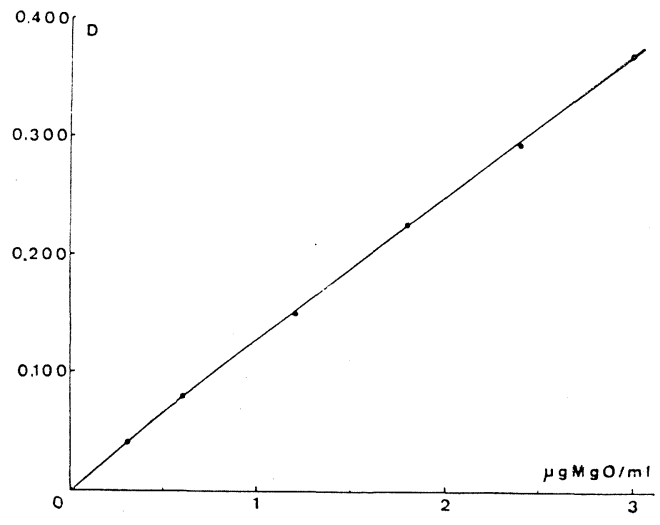

Fig. 3

$\begin{array}{ll}\text { LLAMA } & : \mathrm{C}_{2} \mathrm{H}_{2}-\text { AIRE } \\ \lambda & : 2852 \AA \\ \text { RENDIJA } & : 7 \AA \\ \text { EXP. DE ESCALA }: & 1\end{array}$

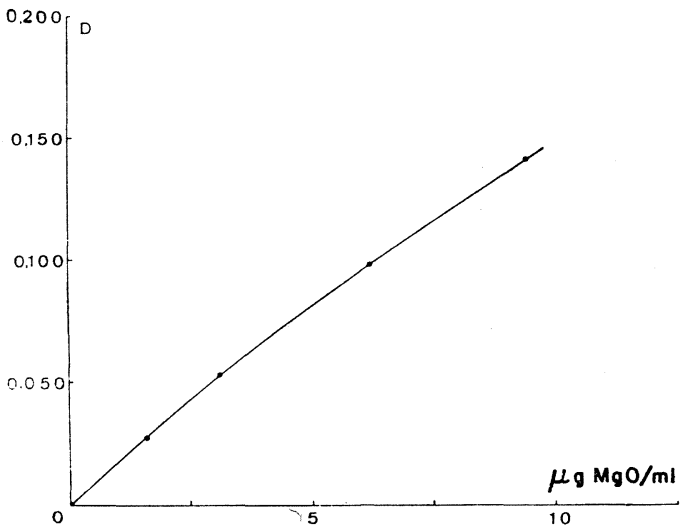

Fig. 4

$\begin{array}{ll}\text { LLAMA } & : \mathrm{C}_{2} \mathrm{H}_{2}-\text { AIRE } \\ \lambda & : 2852 \AA \\ \text { RENDIJA } & : \quad 7 \AA \\ \text { EXP. DE ESCALA }: & 3\end{array}$

Damos más abajo los valores L.W.R. para los diez elementos; estas cifras han sido tomadas de documentos facilitados por Perkin-Elmer (11).

Aquellas cifras han sido calculadas para disoluciones que contienen solamente un elemento; ahora bien, hemos observado que estos datos corresponden perfectamente a las L.W.R. que se encuentran para cada óxido en nuestras disoluciones-patrón.

\begin{tabular}{|c|c|c|c|c|c|c|c|c|c|c|}
\hline Oxidos & $\mathbf{C a O}$ & $\mathrm{SiO}_{2}$ & $\mathrm{Al}_{2} \mathbf{O}_{3}$ & $\mathrm{Fe}_{2} \mathbf{O}_{3}$ & $\mathbf{M g O}$ & $\mathrm{Na}_{2} \mathbf{O}$ & $\mathbf{K}_{2} \mathbf{O}$ & $\mathrm{Mn}_{2} \mathbf{O}_{3}$ & $\mathrm{SrO}$ & $\mathbf{T i O}_{2}$ \\
L.W.R. & 10 & 320 & 100 & 7 & 0,8 & 1,3 & 2,4 & 3,3 & 6 & 330 \\
\hline
\end{tabular}

En las figuras 5 y 6 están las curvas de $\mathrm{D}$ en función de la concentración de $\mathrm{Na}_{2} \mathrm{O}$ (la curva 6 está con expansión de escala). En las figuras 7 y 8 pueden verse las mismas curvas para el $\mathrm{Mn}_{2} \mathrm{O}_{3}$.

Las figuras 9, 10, 11, 12 y 13 expresan las variaciones de $\mathrm{D}$ en función de la concentración de los óxidos siguientes: $\mathrm{Al}_{2} \mathrm{O}_{3}, \mathrm{Fe}_{2} \mathrm{O}_{3}, \mathrm{~K}_{2} \mathrm{O}, \mathrm{TiO}_{2}$ y $\mathrm{SrO}$.

Para todos los elementos que están representados en las curvas $D=f(C)$, de las figuras 3 a la 13, se aplica el método de cálculo que ya hemos descrito. Diremos que no es necesario utilizar todas las disoluciones-patrón para la valoración de un cemento: es suficiente seleccionar aquellas que tengan todos los elementos que se deseen valorar. 


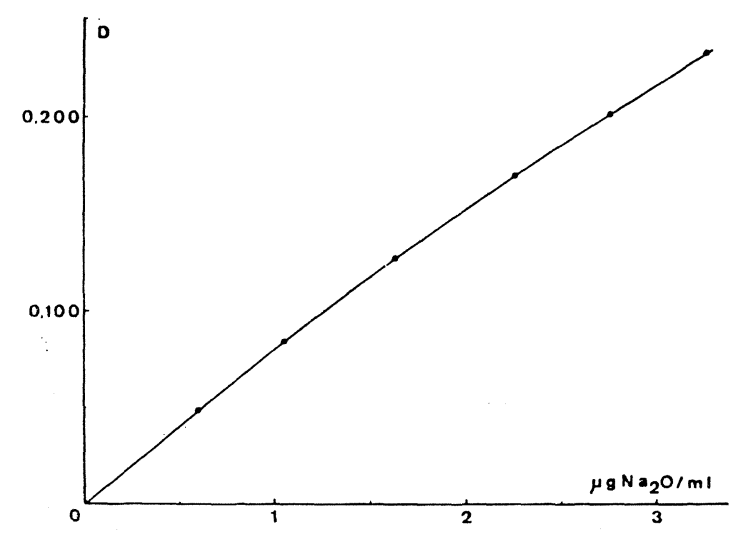

Fig. 5

LLAMA

$\lambda$

RENDIJA

EXP. DE ESCALA : 1

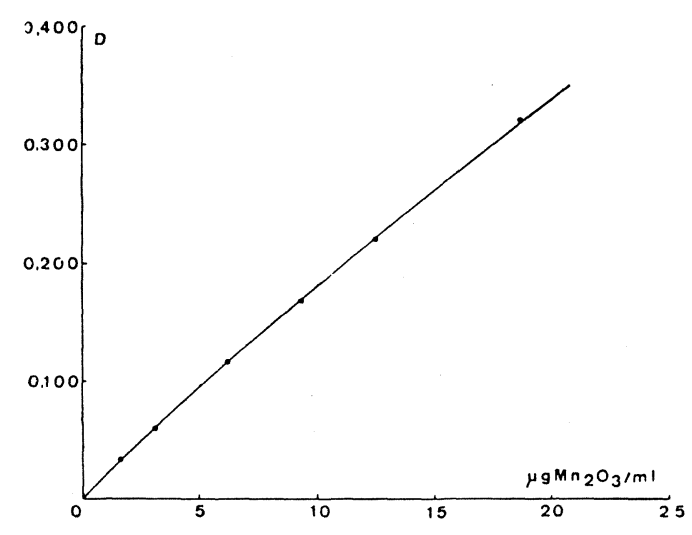

Fig. 7

$\begin{array}{ll}\text { LLAMA } & : \mathrm{C}_{2} \mathrm{H}_{2}-\text { AIRE } \\ \lambda & : 2795 \AA \\ \text { RENDIJA } & : 2 \AA \\ \text { EXP. DE ESCALA } & : 1\end{array}$

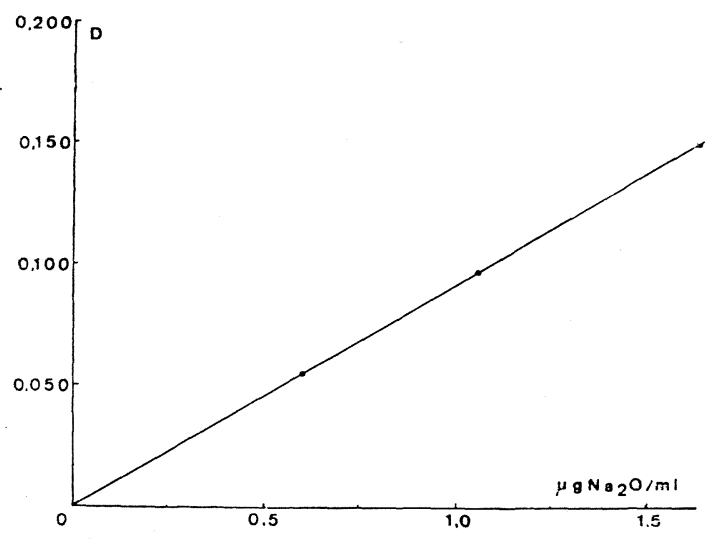

Fig. 6
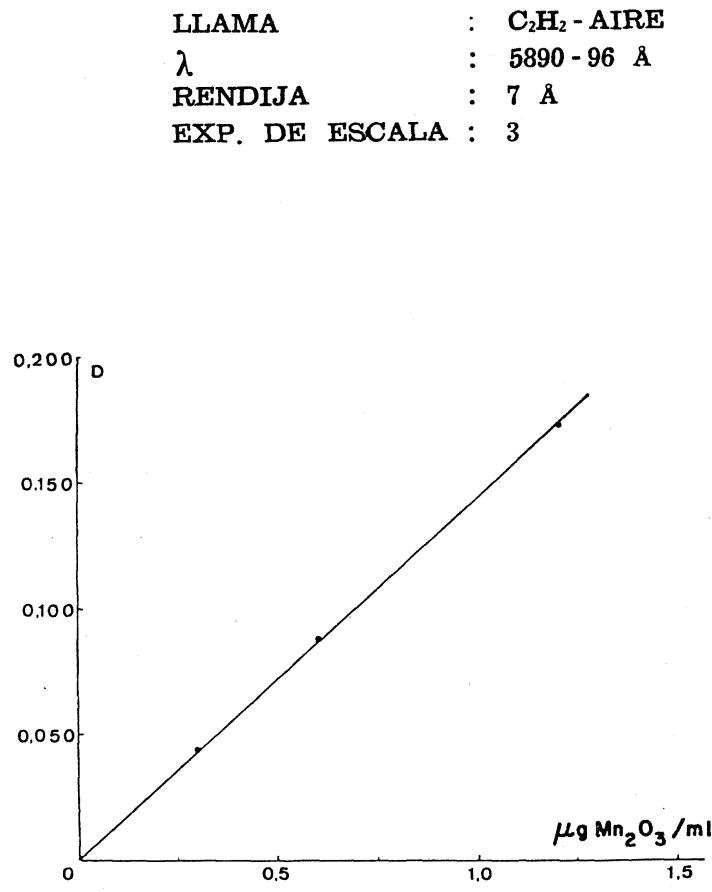

Fig. 8

LLAMA : $\mathrm{C}_{2} \mathrm{H}_{2}-\mathrm{AIRE}$

$\lambda$

RENDIJA : $2 \AA$

EXP. DE ESCALA : 3

Siendo tan elevada la concentración de $\mathrm{CaO}$ y $\mathrm{SiO}_{2}$ en los cementos, las determinaciones de estos óxidos se realizan con expansión de escala y supresión de cero.

La figura 14 muestra la curva $D=f(C)$ para la sílice. En las 15 y 16 figuran las curvas $\mathrm{D}=\mathrm{f}(\mathrm{C})$ para la cal. Las siglas "U.A." que se ven en las figuras 15 y 16 son una abreviatura de "Unidad Arbitraria", que representa, en este caso, las divisiones del papel de registro. 


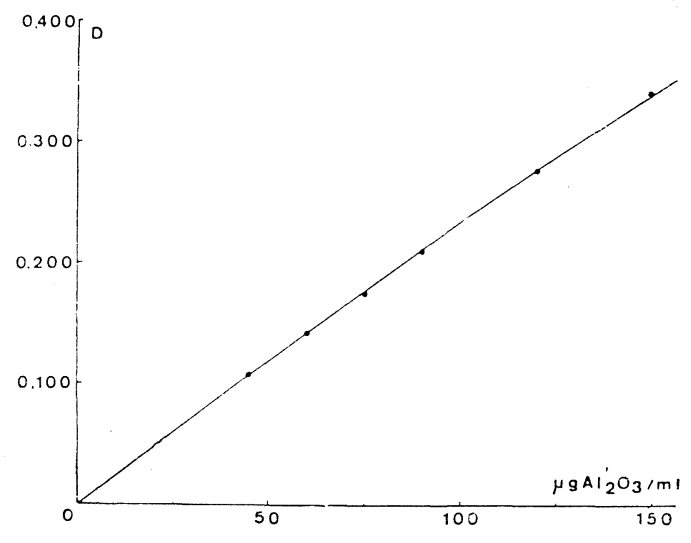

Fig. 9

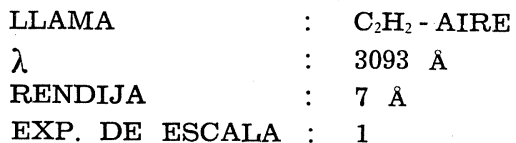

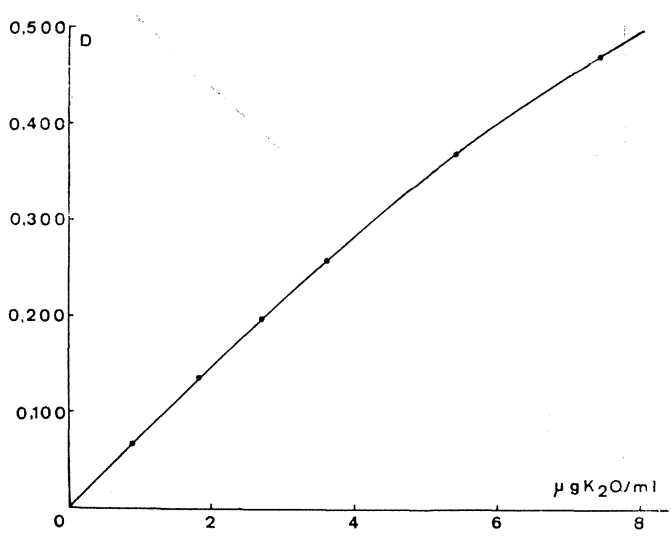

Fig. 11

$\begin{array}{ll}\text { LLAMA } & : \mathrm{C}_{2} \mathrm{H}_{2}-\mathrm{AIRE} \\ \lambda & : 7665 \AA \\ \text { RENDIJA } & : 7 \AA \\ \text { EXP. DE ESCALA } & : 1\end{array}$

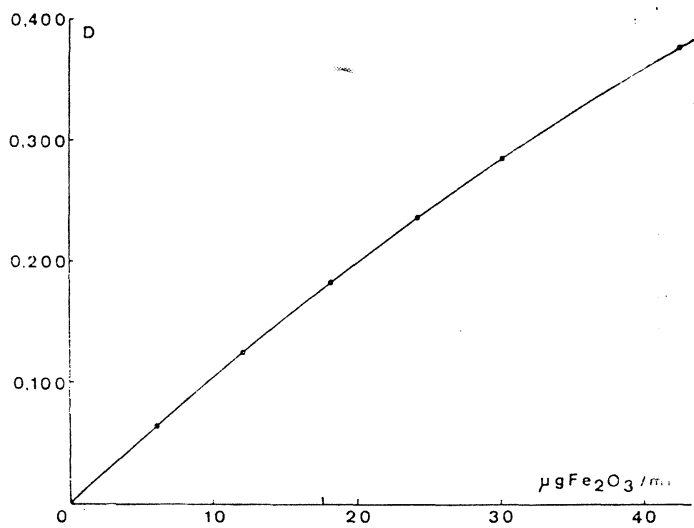

Fig. 10

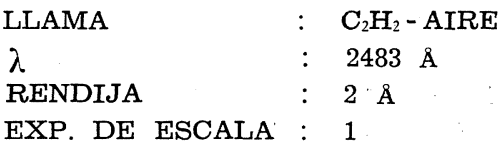

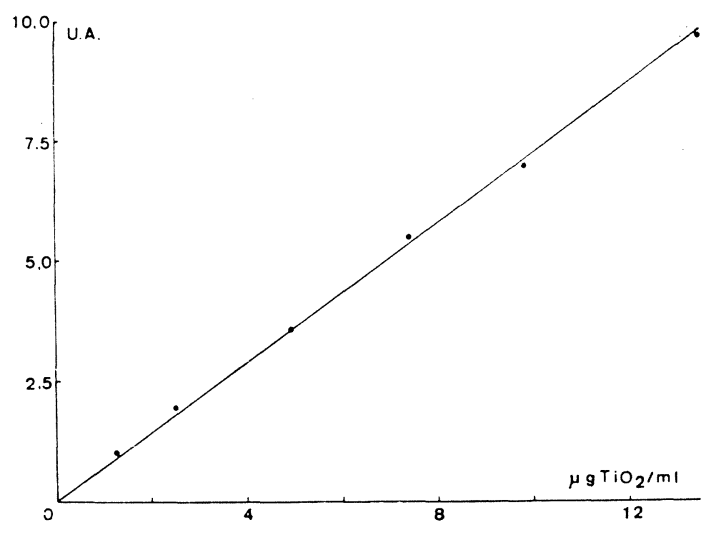

Fig. 12

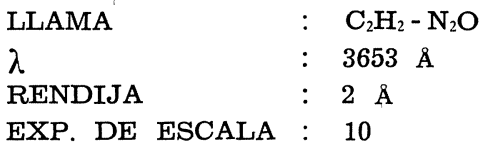

Para el cálculo de la cantidad de $\mathrm{CaO}$ es necesario sustituir la disolución en la que se ignora la concentración, $\mathrm{C}_{\mathrm{x}}$, por dos disoluciones-patrón de concentración, $\mathrm{C}_{\mathrm{e}} \mathrm{y}$ $\mathrm{C}_{\mathrm{e} I I}$. La fórmula dada en la figura 17 permite calcular muy rápidamente las cantidades en $\mathrm{CaO}$, sin recurrir a las densidades ópticas.

Para calcular las cantidades en $\mathrm{SiO}_{2}$ se hace lo mismo que lo que ya hemos esquematizado en la figura 17 para el cálculo de $\mathrm{Ca}$. 


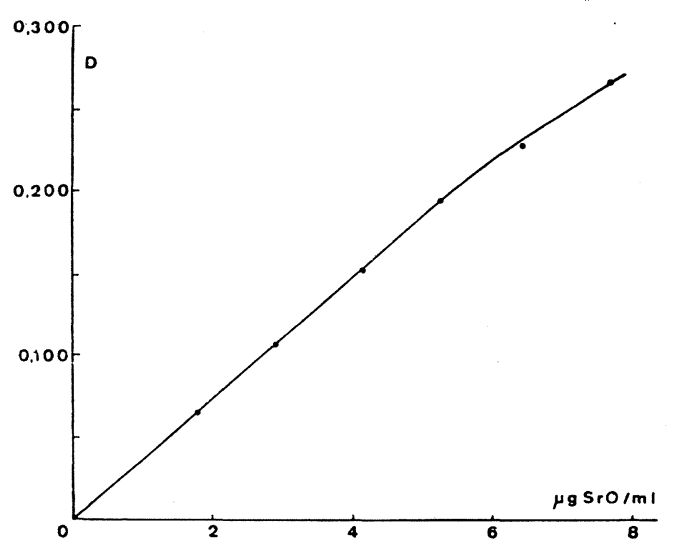

Fig. 13

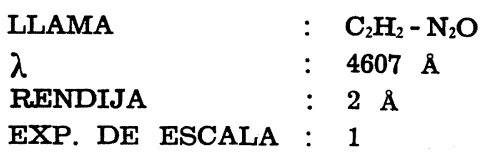

EXP. DE ESCALA : 1

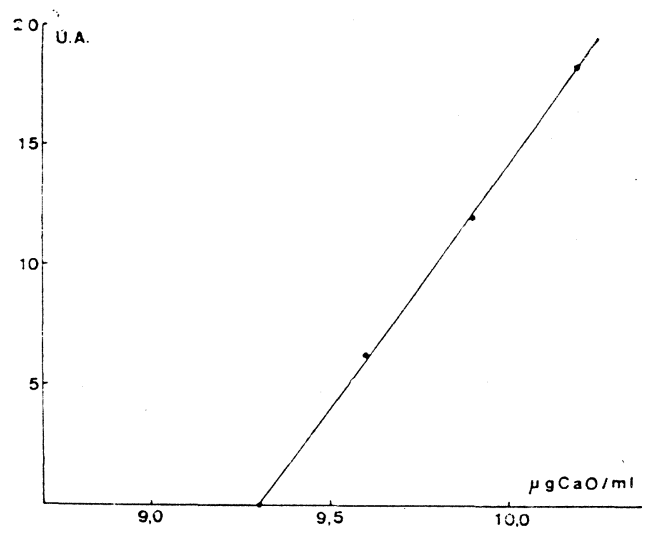

Fig. 15

$\begin{array}{ll}\text { LLAMA } & : \mathrm{C}_{2} \mathrm{H}_{2}-\text { AIRE } \\ \lambda & : 4227 \AA \\ \text { RENDIJA } & : 7 \AA \\ \text { EXP. DE ESCALA } & : 10 \text { sup. } 0\end{array}$

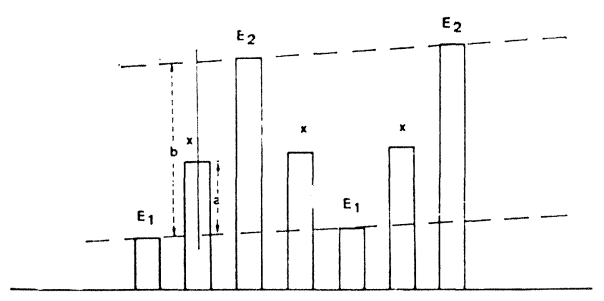

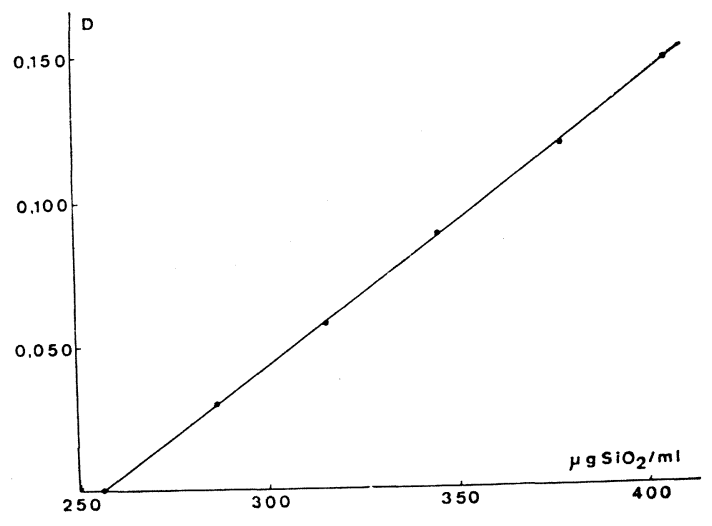

Fig. 14
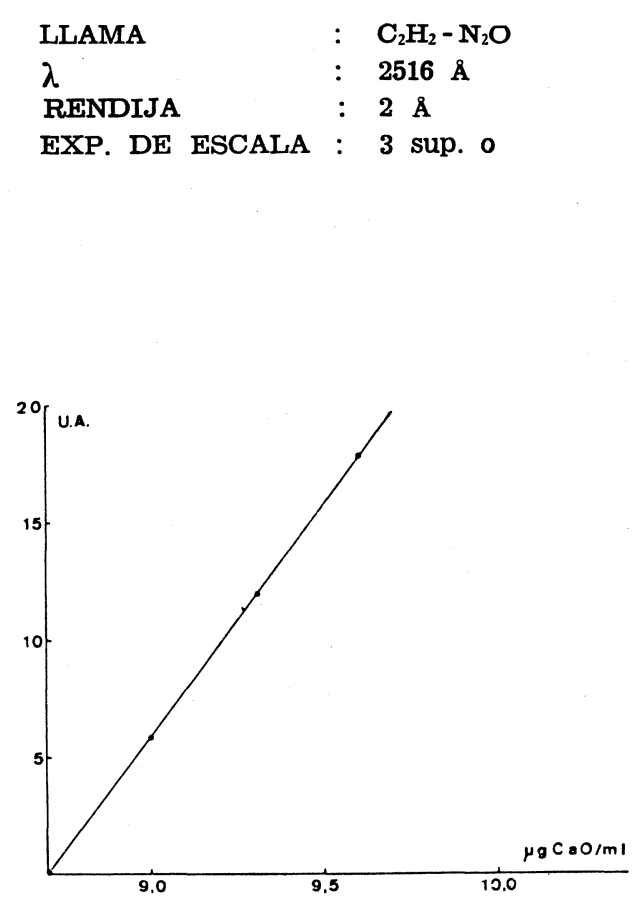

Fig. 16

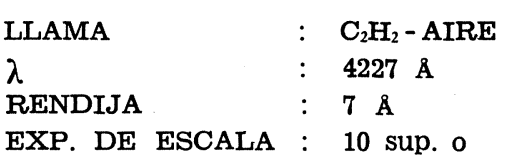

Fig. 17.-Dosificación del Ca

$\mathbf{C}_{\mathrm{x}}=\mathrm{C}_{\mathrm{E} 1}+\frac{\left(\mathrm{C}_{\mathrm{E} 2}-\mathrm{C}_{\mathrm{E} 1}\right) \mathrm{a}}{\mathrm{b}}$

$\lambda \quad: 4227 \AA$

RENDIJA : $7 \AA$

EXP. DE ESCALA : 10 sup. o 


\section{Condiciones instrumentales}

Para la realización de este trabajo se ha utilizado un espectrofotómetro Perkin-Elmer, de doble haz (Modelo 303); se ha utilizado siempre un registrador. El tiempo de registro de las señales es del orden de 15 segundos.

Las características técnicas del espectrofotómetro con el cual se ha trabajado están descritas por Perkin-Elmer (11). Las condiciones óptimas instrumentales -reglaje del nebulizador, altura del mechero, consumo de gas, etc.- se obtuvieron por ensayos preliminares.

Las condiciones instrumentales se relacionan en la tabla 3.

Para todas las determinaciones hemos empleado el mechero de titanio (303-0420), que en principio está indicado para $\mathrm{N}_{2} \mathrm{O}_{-} \mathrm{C}_{2} \mathrm{H}_{2}$. Este quemador se utiliza en posición paralela para la determinación de todos los elementos.

\section{Resultados y conclusiones}

Se han analizado por este método dos cementos N.B.S.: los resultados se resumen en la tabla 4 .

El examen de esta tabla demuestra que la exactitud del método es completamente satisfactoria. Las desviaciones entre los valores encontrados por la E.A.A. y los reales son insignificantes. A nuestro entender, es la primera vez que se da con precisión por E.A.A. diez elementos partiendo tan sólo de una disolución de la muestra.

Roos y Price (12) han intentado dosificar todos los elementos de un cemento practicando una sola disolución. El método de la disolución del cemento que han realizado se parece al nuestro: está basado en el ataque del cemento por una mezcla de $\mathrm{HCl}+\mathrm{HF}$.

La precisión en la valoración de los óxidos, exceptuando el $\mathrm{CaO}$ y el $\mathrm{SiO}_{2}$, es el mismo en nuestro caso y en el de Roos y Price, pero a estos investigadores les sale una desviación en el valor de $\mathrm{Ca}$ y $\mathrm{Si}$ de 0,7 y $0,24 \%$, respectivamente, mientras que por nuestro método son de 0,08 y $0,09 \%$, respectivamente.

La precisión de nuestros métodos es, en general, superior al obtenido por métodos clásicos (volumetría y gravimetría). Desde el punto de vista de la rapidez, es muy superior al de la complexometría o al de la gravimetría.

Si se compara la E.A.A. con la fluorescencia de rayos X, se puede decir, por lo que hasta ahora se sabe, que la E.A.A. no puede reemplazar a la fluorescencia en el control automático de fabricación. Pero si no se practica ese control automático, la E.A.A. es mucho más interesante que la fluorescencia de rayos X.

Comparando la E.A.A. con los equipos de rayos $\mathrm{X}$ perfeccionados, es necesario tener en cuenta que una instalación multicanal de rayos $\mathrm{X}$, para valoraciones simultáneas de muchos elementos, es, con mucho, más costosa que el espectrofotómetro de absorción atómica más perfeccionado que exista. 
T A B L A 3

Condiciones experimentales

\begin{tabular}{|c|c|c|c|c|c|c|c|c|c|c|}
\hline Elemento & $\begin{array}{l}\text { Longitud } \\
\text { de onda } \\
\lambda \text { en } \AA\end{array}$ & $\begin{array}{c}\text { Anchura } \\
\text { de rendija } \\
\text { en }-\AA\end{array}$ & $\begin{array}{c}\text { Intensidad } \\
\text { de la } \\
\text { Iámpara } \\
\mathbf{m A}\end{array}$ & $\begin{array}{c}\text { Altura } \\
\text { del quemador } \\
\text { en } \mathrm{mm}\end{array}$ & $\begin{array}{l}\text { Mezcla } \\
\text { gaseosa }\end{array}$ & $\begin{array}{c}\text { Consumo } \\
\text { carburante } \\
l / \text { min }\end{array}$ & $\begin{array}{c}\text { Consumo } \\
\text { combustible } \\
l / \text { min }\end{array}$ & $\begin{array}{l}\text { Naturaleza } \\
\text { de la } \\
\text { llama }\end{array}$ & $\begin{array}{l}\text { Expansión } \\
\text { de escala }\end{array}$ & $\begin{array}{l}\text { Constante } \\
\text { de tiempo }\end{array}$ \\
\hline Calcio & 4.227 & 7 & 10 & 8 & Aire- $\mathrm{C}_{2} \mathrm{H}_{2}$ & 16 & 2,5 & Oxidante & $\times 10$ & 3 \\
\hline Silicio & 2.516 & 2 & 40 & 9 & $\mathrm{~N}_{2} \mathrm{O}-\mathrm{C}_{2} \mathrm{H}_{2}$ & 12 & 7 & $\begin{array}{l}\text { Reductora } \\
\text { cono rosa } 4 \mathrm{~cm}\end{array}$ & $\mathrm{x} 3$ & 3 \\
\hline Aluminio & 3.093 & 7 & 25 & 8 & $\mathrm{~N}_{2} \mathrm{O}-\mathrm{C}_{2} \mathrm{H}_{2}$ & 14,5 & 8 & $\begin{array}{l}\text { Reductora } \\
\text { cono rosa } 2 \mathrm{~cm}\end{array}$ & $\begin{array}{ll}x & 1\end{array}$ & 2 \\
\hline Hierro & 2.483 & 2 & 30 & 7,5 & Aire- $\mathrm{C}_{2} \mathrm{H}_{2}$ & 16 & 2,5 & Oxidante & $\mathrm{X} \quad 1$ & 2 \\
\hline Magnesia & 2.852 & 7 & 6 & 9 & Aire- $\mathrm{C}_{2} \mathrm{H}_{2}$ & 16 & 2,5 & Oxidante & $\begin{array}{ll}\mathrm{X} & 1 \\
\mathrm{X} & 3\end{array}$ & $\begin{array}{l}2 \\
3\end{array}$ \\
\hline Sodio & $\begin{array}{l}5.890 \\
5.896\end{array}$ & 7 & 10 & 10 & Aire- $\mathrm{C}_{2} \mathrm{H}_{2}$ & 16 & 2,5 & Oxidante & $\begin{array}{ll}X & 1 \\
X & 3\end{array}$ & $\begin{array}{l}2 \\
3\end{array}$ \\
\hline Potasio & 7.665 & 7 & 12 & 9 & Aire- $\mathrm{C}_{2} \mathrm{H}_{2}$ & 16 & 2,5 & Oxidante & $\begin{array}{ll}x & 1\end{array}$ & 3 \\
\hline Manganeso & 2.795 & 2 & 20 & 11 & Aire $\mathbf{C}_{2} \mathrm{H}_{2}$ & 16 & 3,5 & Reductora & $\begin{array}{ll}\mathrm{X} & 1 \\
\mathrm{X} & 3\end{array}$ & $\begin{array}{l}2 \\
3\end{array}$ \\
\hline Estroncio & 4.607 & 2 & 20 & 6 & $\mathrm{~N}_{2} \mathrm{O}-\mathrm{C}_{2} \mathrm{H}_{2}$ & 10 & 3 & $\begin{array}{l}\text { Reductora } \\
\text { cono rosa } 0,5 \mathrm{~cm}\end{array}$ & $\begin{array}{ll}\mathrm{X} & 1\end{array}$ & 3 \\
\hline Titanio & 3.653 & 2 & 40 & 10 & $\mathrm{~N}_{2} \mathrm{O}-\mathrm{C}_{2} \mathrm{H}_{2}$ & 12 & 7 & $\begin{array}{l}\text { Reductora } \\
\text { cono rosa } 4 \mathrm{~cm}\end{array}$ & X 10 & 3 \\
\hline
\end{tabular}


T A B L A 4

\begin{tabular}{|c|c|c|c|c|c|c|}
\hline Oxido & $\begin{array}{c}\text { NBS } 1013 \\
\text { valor certificado } \\
\text { en } \%\end{array}$ & \begin{tabular}{|c|} 
NBS 1013 \\
valor encontrado \\
por la E.A.A. \\
en $\%$
\end{tabular} & $\begin{array}{c}\text { Desviación } \\
\text { típica } \\
\sigma \%\end{array}$ & $\begin{array}{c}\text { NBS } 1014 \\
\text { valor certificado } \\
\text { en } \%\end{array}$ & \begin{tabular}{|c} 
NBS 1014 \\
valor encontrado \\
por la E.A.A. \\
en $\%$
\end{tabular} & $\begin{array}{c}\text { Desviación } \\
\text { típica } \\
\sigma \%\end{array}$ \\
\hline $\mathrm{CaO}$ & 64,26 & 64,19 & 0,08 & 63,10 & 63,03 & 0,11 \\
\hline $\mathrm{SiO}_{2}$ & 24,17 & 24,00 & 0,11 & 19,49 & 19,40 & 0,09 \\
\hline $\mathrm{Al}_{2} \mathrm{O}_{3}$ & 3,30 & 3,306 & 0,049 & 6,38 & 6,224 & 0,049 \\
\hline $\mathrm{Fe}_{2} \mathrm{O}_{3}$ & 3,07 & 2,959 & 0,018 & 2,50 & 2,458 & 0,020 \\
\hline MgO & 1,39 & 1,382 & 0,009 & 2,80 & 2,802 & 0,011 \\
\hline $\mathrm{Na}_{2} \mathrm{O}$ & 0,20 & 0,1965 & 0,0019 & 0,24 & 0,2427 & 0,0023 \\
\hline $\mathrm{K}_{2} \mathrm{O}$ & 0,32 & 0,3274 & 0,0022 & 0,99 & 0,9777 & 0,0062 \\
\hline $\mathrm{Mn}_{2} \mathrm{O}_{3}$ & 0,05 & 0,0454 & 0,0008 & 0,07 & 0,0644 & 0,0009 \\
\hline $\mathrm{SrO}$ & 0,08 & 0,0844 & 0,0008 & 0,26 & 0,2572 & 0,0033 \\
\hline $\mathrm{TiO}_{2}$ & 0,20 & 0,2180 & 0,0065 & 0,25 & 0,2544 & 0,0095 \\
\hline R.I. & - & 0,12 & - & - & 0,15 & 一 \\
\hline
\end{tabular}

En la industria vidriera, donde la preparación de muestras no presentan ningún problema para la técnica de rayos X (13), a veces se emplea la absorción atómica como comprobación de patrones de fluorescencia.

El método por nosotros propuesto permite determinar rápidamente diez óxidos en un cemento. Es muy interesante cuando se deba conocer el análisis completo del cemento. En los laboratorios de control, corrientemente es necesario valorar tan sólo los cuatro óxidos $-\mathrm{CaO}, \mathrm{SiO}_{2}, \mathrm{Al}_{2} \mathrm{O}_{3}$ y $\mathrm{Fe}_{2} \mathrm{O}_{3}$ - para calcular el índice de saturación de cal.

Desde luego, en este tipo de análisis se necesita una precisión aceptable; pero un factor a tener muy en cuenta es la rapidez.

Butler y Kröger (14) han examinado recientemente las posibilidades de la absorción atómica para el control de fabricación del cemento. Estos autores se han dedicado a valorar con la mayor rapidez posible los cuatro óxidos citados. En el caso más favorable, según ellos, la dosificación de estos cuatro óxidos necesita sólo 30 minutos, pero, en nuestra opinión, la de la cal no es bastante precisa.

Adoptando el método que proponemos, para la valoración rápida de 4 ó 5 óxidos (en lugar de diez), se podrá, sin duda, disminuir el tiempo de análisis, manteniendo, además, una excelente precisión, sobre todo para la determinación de la cal.

Diremos, a este respecto, que los espectrofotómetros modernos permiten obtener directamente la concentración.

El empleo de estos aparatos suprime el tiempo empleado en el cálculo de los resultados; intentamos, asimismo, simplificar las diluciones. De momento, efectuamos 5 diluciones para la determinación de diez óxidos: sería interesante valorar los cuatro óxidos 
- $\mathrm{CaO}, \mathrm{SiO}_{2}, \mathrm{Al}_{2} \mathrm{O}_{3}$ y $\mathrm{Fe}_{2} \mathrm{O}_{3}$ - practicando dos diluciones. Nuestros primeros resultados son alentadores; por otra parte, investigamos el método de simplificar las disolucionespatrón.

En conclusión, se puede decir que la absorción atómica no solamente es de interés para la determinación de la magnesia o de los óxidos minoritarios $-\mathrm{Na}_{2} \mathrm{O}, \mathrm{K}_{2} \mathrm{O}, \mathrm{Mn}_{2} \mathrm{O}_{3}$, $\mathrm{SrO}$, etc.- sino que permite también la valoración rápida de los óxidos mayoritarios, con una precisión, por lo menos, igual a la de la gravimetría.

\section{B I B L I O G R A F I A}

(1) N. Tenoutasse, A. DE Donder, R. Smitz.: Silicates Industriels, Tome XXXV, n. $1,1970$.

(2) N. Tenoutasse.: Revue des Matériaux, Ciments et Bétons. 1. ${ }^{\text {re }}$ partie: n. ${ }^{\circ}$ 664-665, 1971 - 2. partie, n. $666,1971-3 .^{e}$ partie: n. $668,1971$.

(3) N. Tenoutasse.: 3. ${ }^{\text {e }}$ Congrès International de Spectrométrie d'absorption et de Fluorescence Atomique (C.I.S.A.F.A.) 27 septembre au 1 . $^{\text {er }}$ octobre. Paris 1971.

(4) F. Bonomi, M. Francardi et F. Massazza.: Il Cemento, Vol. 68, n. 2 2, avril-juin 1971 , p. 65.

(5) I. VoInivitch, G. Legrand et J. LouvrIer.: 3. C.I.S.A.F.A. 27 septembre-1. ${ }^{\text {er }}$ octobre 1971 , Paris.

(6) I. VoINovitch.: Communication personelle.

(7) I. Rubeck \& B. Moldan.: Anal. Chim. Acta 37 (1967) 421.

(8) C. Riandey.: Chimie Analytique, Vol. 53 n. 7439 (1971).

(9) T. C. RaIns.: Flame Emission and Atomic Absorption Spectrometry. Ed. by J. A. DEAN and T. C. RAINS, Vol. 1, page 349 .

(10) B. Bernas, : Anal. Chem. 11 (1968) 1683.

(11) Analytical Methods for Atomic Absorption Spectrophotometry. March. 71 PERKIN-ELMER, Norwalk, Connecticut, U.S.A.

(12) J. T. H. Roos et W. J. PrICE. : Analyst Vol. 44 (1969) 89.

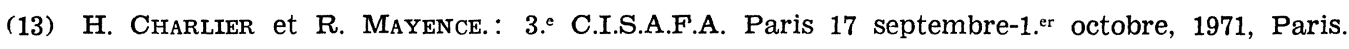

(14) L. R. P. Butler et K. KRöger.: Cement Technology may/june 1971. 